\title{
A New feature extraction method to Improve Emotion Detection Using EEG Signals
}

\author{
Hanieh Zamanian, Hassan Farsi \\ Department of Electrical and Computer engineering, University of Birjand, Birjand, Iran
}

Received 14th Jan 2017; accepted 22 Jul 2018

\begin{abstract}
Since emotion plays an important role in human life, demand and importance of automatic emotion detection have grown with increasing role of human computer interface applications. In this research, the focus is on the emotion detection from the electroencephalogram (EEG) signals. The system derives a mechanism of quantification of basic emotions using. So far, several methods have been reported, which generally use different processing algorithms, evolutionary algorithms, neural networks and classification algorithms. The aim of this paper is to develop a smart method to improve the accuracy of emotion detection by discrete signal processing techniques and applying optimized support vector machine classifier with genetic evolutionary algorithm. The obtained results show that the proposed method provides the accuracy of 93.86\% in detection of 4 emotions (happy, sad, exiting and hate) which is higher than state-of-the-art methods.
\end{abstract}

Keywords: emotion recognition, EEG, Arousal-Valence emotion model, support vector machine, neural

network.

\section{Introduction}

Human brain may be considered as a computer with electrochemical process, which converts sensory information into electrical information (voltage). Emotions are complex set of guidelines, which reflect the changes in human body. A variety of emotions such as anger, depression, despair, hope, hate, fear, sadness, surprise, happiness and etc. have been identified, which are used to model a system for emotion detection. In recent years, research based on emotion recognition from EEG has attracted great interest from a vast amount of interdisciplinary fields in psychology to engineering, including basic studies on emotion theories and applications to affective BrainComputer Interaction (ABCI) (Mühl et al., 2014)[1]. This enhances the BCI systems with the ability to detect, process, and respond to affective states using physiological signals.

Correspondence to: < hfarsi@birjand.ac.ir >

Recommended for acceptance by Josep LIadós

https://doi.org/10.5565/rev/elcvia.1044

ELCVIA ISSN: $1577-5097$

Published by Computer Vision Center / Universitat Autonoma de Barcelona, Barcelona, Spain 
Emotion variation of an individual cause significant impact on the brain signals. So, The Electroencephalogram (EEG) signals which reflect the electrical information of the brain are highly regarded during the recent years. The EEG recording devices, depending on the application, use 3 to 256 electrodes placed on the scalp to record the relative voltages. For clinical applications 8 to 32 EEG channels are usually required.

For incidence of different emotions, it is possible to use music, text, video and images, to provoke individuals and therefore, the emotion signals can be recorded. Then the recorded signals, as the database are used in recognition algorithms.

Diagnostic procedures are distinct based on the extracted features and different classification algorithms. In case of the EEG signal, selection of the number, and type of channels, can be considered as an identification parameter.

Many features can be derived from EEG signals. These features are obtained by applying different transformations on the EEG signals. For example, Fourier transform focuses on a specific frequency bands.

The classification task sets the boundaries of the decisions in nonlinear optimal way. So far, different classification methods for emotion detection based on EEG signals have been reported such as SVM, KNN and neural network classifiers, cited as the most widely used techniques.

This paper is organized as follows: In Section 2, a brief systematic overview of brain signal analysis methods and classification procedure for feature extraction, and classifiers are identified. Section 3 details all required materials and protocols. We use time-frequency analysis to find appropriate features for different emotions, and then evaluate our emotion recognition model over time on the DEAP dataset. In Section 4, the experimental results are presented and compared to other methods. Finally, the conclusion is drawn in section 5.

\section{Related works}

In emotion recognition, a vast amount of studies has been conducted for effective computing based on different signals. Many efforts have been made to recognize emotions using external emotion expression, such as facial expression [3] and speech [4]. However, some times the emotional states remain internal and cannot be detected by external expression. For instance, consider extreme cases where people do not say anything but actually they are angry, even smiley during negative emotional states due to the social masking. In these cases, the external emotional expression can be controlled subjectively and these external cues for emotion recognition may be inadequate [2].

Emotional experience associates with a particular pattern of physiological activity. Unlike audiovisual expression of feelings, using physiological activity is more objective and easier. The physiological activities can be achieved through non-invasive sensors. These sensors receive and record the electrical signals representing EEG signals which can be displayed. Many studies on construction of computational models to estimate the emotional state of the EEG signal are provided which indicated the efficiency of these models to detect the emotions. They used several incentives to stimulate feelings with photos, music and videos. The feelings examined in many studies are discrete.

Most of the studies are based on two steps, first is feature extraction and second, is to choose and apply a classification algorithm. In the following we focus on different features extracted from EEG signals for emotion detection. Some of these features are extracted in time domain of EEG 
signals while others achieved in frequency domain. Therefore, we divide the features in two parts as follows.

\section{a) Time domain features}

These features are extracted from the signal in its original domain. First of all, the statistical properties of the signal such as mean, standard deviation and power [6-9] are mostly used. Another feature is entropy, which is the symbol of scattering data. However as shown in [10], the change in information entropy within the EEG signal may window a real change in cortical functional organization. Thus, the term 'entropy' may be more useful than merely a statistical measure of EEG pattern, but in some cases reflect the intra-cortical information flow. Also, Hjorth in 1970 [11], introduced some features called activity, mobility and complexity, which are then used by researchers for EEG emotion detection $[12,13]$. Other common features in time domain include fractal dimension [14], non-stationary index [15], Higher Order Crossings [16, 17]. More explanation about these features could be found in [5].

\section{b) Frequency domain features}

Presentation of a signal in frequency domain is possible by using Fourier transform. The mostly used algorithm to compute Discrete Fourier Transform (DFT) is Fast Fourier Transform (FFT). The most popular feature in the context of emotion recognition from EEG is power in different frequency bands $[19,20]$. It is assumed that the signal is stationary for the duration of a trial. Different frequency bands are found by wavelet transform. The frequency bands of EEG signals slightly vary in different studies. Table 1 shows the frequency bands with relative bandwidth and the level of wavelet decomposition. The extracted features from the resulting representation of the signal in frequency domain are: average power (mean) of frequency bands [21-23], and relative minimum, maximum, and variance. Additionally, the ratio of mean band powers is calculated for each channel. A set of frequency domain features has been introduced by Hosseini and et al. in [18] which is called higher order spectra.

Table1. Frequency bands with relative bandwidth and decomposition levels of EEG signals recorded at $\mathrm{fs}=256 \mathrm{~Hz}$

\begin{tabular}{|c|c|c|}
\hline Bandwidth $(\mathrm{Hz})$ & Frequency band & Level of Wavelet decomposition \\
\hline $1-4$ & $\delta$ & A6 \\
\hline $4-8$ & $\theta$ & D6 \\
\hline $8-10$ & $\alpha$ slow & D5 $(8-16 \mathrm{~Hz})$ \\
\hline $10-12$ & $\alpha$ & $\mathrm{D} 4(16-32 \mathrm{~Hz})$ \\
\hline $12-30$ & $\beta$ & $\mathrm{D} 3(32-64 \mathrm{~Hz})$ \\
\hline $30-64$ & $\gamma$ & \\
\hline
\end{tabular}

\section{c) Time frequency domain features}

If a signal is non-stationary, time-frequency methods can provide additional information by considering dynamical changes [5]. In this category we can mention the features extracted by applying the wavelet transform to the signal [24, 25]. Also, Hilbert- Huang spectrum of intrinsic mode functions is used [26]. 
The feature extraction methods related to other studies are presented in Table 2. After feature extraction, the extracted features should be classified by using an appropriate classification algorithm. Many algorithms have been reported for classification, among them $\mathrm{K}$ Nearest Neighbors (KNN), Support Vector Machine (SVM), Linear Discriminant Analysis (LDA), Quadratic Discriminant Analysis (QDA) and Neural network are mostly used. Some researches comprise several of these classification algorithms and most of them show the SVM-based algorithm provides better performance [34].

Table2. Some feature extraction methods used for EEG emotion detection

\begin{tabular}{|c|c|c|c|c|}
\hline Author & Year & Feature extraction method & $\begin{array}{c}\text { Number } \\
\text { of } \\
\text { classes }\end{array}$ & Accuracy(\%) \\
\hline Bajaj and Pachori [27] & 2015 & Multiwavelet & 4 & 84.79 \\
\hline Lin and Sourina [28] & 2014 & $\begin{array}{c}\text { HOC, statistical and fractal } \\
\text { dimension }\end{array}$ & 40 \\
\hline Park et al. [29] & 2013 & $\begin{array}{c}\text { Power of each frequency band, } \\
\text { celebral asymmetry and } \\
\text { coherence }\end{array}$ & 3 & 66.3 \\
\hline Kwon et al. [30] & 2013 & $\begin{array}{c}\text { Power difference between right } \\
\text { and left hemispheres in alpha and } \\
\text { gamma band }\end{array}$ & 2 & 64.78 \\
\hline Chung and Yoon [31] & 2012 & Power spectral density & 3 & 52.2 \\
\hline $\begin{array}{c}\text { Hosseini and } \\
\text { Khalilzadeh [32] }\end{array}$ & 2010 & Wavelet and Fractal dimension & 2 & 79.20 \\
\hline $\begin{array}{c}\text { Khosrowabadi and } \\
\text { Roham [33] }\end{array}$ & 2010 & Fractal dimension & 4 & 81 \\
\hline
\end{tabular}

\section{Methodology}

In reported methods, the aim is to create an efficient method to find appropriate features and classifier. This research focuses on both feature extraction and classifier and tries to improve the emotion detection from the brain's signals. First of all, the number of EEG channels which will be later used in feature extraction procedure is required to be identified. Next, feature extraction procedure is applied on the database signals. Finally, the extracted features are classified and the efficiency of the proposed method is then evaluated.

\subsection{Database}

The proposed algorithm is tested on a benchmark multimodal dataset known as the DEAP dataset which is prepared by recording EEG signals of 32 participants while watching 40 different kinds of music videos. In this dataset, the data was acquired with a 32 channel BioSemi acquisition system. Figure 1, shows the location of the electrodes on the scalp. All people who participated were healthy, aged between 19 and 32 and also half of the total participants were female. These music videos, used as stimuli, were classified into different emotions utilizing the affective tags [35]. Participants rated each video in terms of the levels of arousal, valence, like/dislike, dominance and familiarity. EEG signals of the participants were down sampled to $128 \mathrm{~Hz}$ before using. Note that the website: Http://www.eecs.qmul.ac.uk/mmv/datasets/deap allows loading the data in appropriate format for MATLAB. 


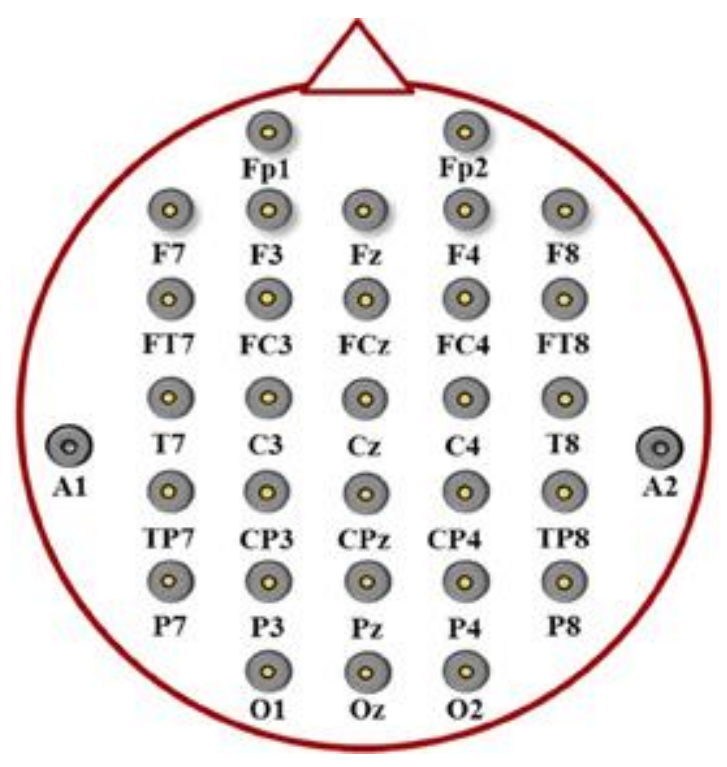

Figure 1. Location of 32 electrodes on the scalp

\subsection{Channel selection}

As mentioned in sub section 3.1, the dataset used for testing the proposed algorithm contains 32 channel data, which is available in 63 seconds with frequency of $128 \mathrm{~Hz}$. So, there is a huge amount of data for each person to be processed. This causes some problems: first, the huge amount of data needs a huge storage memory and second, the procedure of processing requires a long time. Because of these two disadvantages, we decided to reduce the amount of data whereas there is no declination in the classification accuracy. For the data used in this research, we found 7.5 second data is appropriate for convenient feature extraction in our proposed algorithm. Although this causes a great reduction of the data to be processed, however it can be even lower if we choose some special channels and use just their data.

If the algorithm is developed for real-time applications, the required time for feature extraction and the number of channels used should be minimized. If more electrodes are used, the comfort level of the user who wears the device is affected as well. Thus, our main objective is to propose an algorithm performing with adequate accuracy in real-time applications [34].

Different methods have been reported to determine the most effective channels, mainly based on trial and error or heuristic optimization algorithms, [14, 34, 36, 37]. In this research, according to the results of the reported methods two groups of channels are compared. One group contains 3 channels including P7, P3and PZ, and the other contains 7 channels including P7, P3, PZ, PO3, O1, $\mathrm{CP} 2$ and $\mathrm{C} 4$. Thus, the amount of data is greatly reduced.

\subsection{Feature extraction}

The proposed feature extraction method includes 2 general parts: Gabor wavelet features and intrinsic mode functions features. In following, each part is explained.

\section{A) Gabor feature extraction}

Gabor filters (sometimes also called Gabor wavelets or kernels) have shown as a powerful tool for feature extraction. They represent band-limited filters with an optimal localization in the spatial 
and frequency domains. In the spatial domain, a family of 2D Gabor filters can be defined as follows [38]:

$$
\varphi(x \square y)=\frac{f_{u}{ }^{2}}{\pi \gamma \eta} \exp \left(-\left(\frac{f^{2}}{\gamma^{2}} x^{\prime 2}+\frac{f^{2}}{\eta^{2}} y^{\prime 2}\right)\right) \exp \left(j 2 \pi f_{u} x^{\prime}\right)
$$

where $x^{\prime}=x \cos \theta_{v}+y \sin \theta_{v}, y^{\prime}=-x \sin \theta_{v}+y \cos \theta_{v}, f_{u}=f_{\max } / 2^{(u / 2)}$, and $\theta_{v}=v \frac{\pi}{8}$.

Each filter represents a Gaussian kernel function modulated by a complex plane wave whose center frequency and orientation are defined by the parameters $f_{u}$ and $\theta_{v}$, respectively. The parameters $\gamma$ and $\eta$ determine the ratio between the center frequency and the size of the Gaussian envelope and, when set to a fixed value, these parameters ensure that Gabor filters of different scales and a given orientation behave as scaled versions of each other. It has to be noted at this point that with fixed values of the parameters $\gamma$ and $\eta$ the scale of the given Gabor filter is defined uniquely by its center frequency $f_{u}$. Commonly the values of the parameters $\gamma$ and $\eta$ are set to $\sqrt{2}$. The last parameter of the Gabor filters, $f_{\max }$, denotes the maximum frequency of the filters and is commonly set to 0.25 . Researchers typically use Gabor filters with four or five scales and six or eight orientations, i.e., $\mathrm{u}=$ $1,2, \ldots, p-1$ and $v=1,2, \ldots, r-1$, where $\mathrm{p}=4$ or 5 and $\mathrm{r}=6$ or 8 , which results in a filter bank of 24 or 40 Gabor filters [39]. Simulation of the Gabor wavelet contains two steps: 1- construction of Gabor filters with mathematical formula. 2- Convolution the filters with a two dimensional matrix.

In this paper, the matrix which has to be convolved with the Gabor filter bank is constructed by the data of each selected channel in each row. The next step is extraction of appropriate features from constructed matrices after the convolution. In this study, three features including energy, mean amplitude and oriented Gabor phase congruency pattern (OGPCP) are used and detailed as follows:

\section{a) Energy}

Due to the convolution of Gabor filters and creation of two-dimensional matrix, there are two dimensional matrices equal to the number of filters as outputs. Two-dimensional Fourier transform for each output matrix is needed for calculation of energy. For example, if Gabor filters are calculated by 5 scales and 8 different orientations, we have 40 filters totally, which are then convolved with the EEG signal. Therefore, 40 matrices are constructed, and their energies are used as features.

\section{b) Mean amplitude}

This feature is obtained by calculating the mean of the total range of output matrix elements.

\section{c) Oriented Gabor phase congruency pattern(OGPCP)}

Gabor magnitude varies slowly with the spatial position unlike the (Gabor) phase which contains as known, very different values even if it is sampled at image locations only a few pixels apart. This causes that it is difficult to extract reliable and discriminative features from the phase responses. The reason is that most of the existing methods use only the (Gabor) magnitude to construct the Gabor feature vector [40]. To overcome this problem, in [39] a new approach for extracting these features was reported called OGPCP. As our knowledge, these features have not been used for EEG emotion recognition so far. For output matrix, $G_{u \square v}(x \square y)$, the OGPCPs are calculated as:

$$
\operatorname{OGPCP}_{v}(x \square y)=\frac{\sum_{u=1}^{\rho-1} A_{u \square v}(x \square y) \Delta \varphi_{u \square v}(x \square y)}{\sum_{u=1}^{\rho-1}\left(A_{u \square v}(x \square y)+\epsilon\right)}
$$

In which: 


$$
\begin{gathered}
A_{u \square v}(x \square y)=\sqrt{\left(\operatorname{Re}\left(G_{u \square v}(x \square y)\right)\right)^{2}+\left(\operatorname{Im}\left(G_{u \square v}(x \square y)\right)\right)^{2}} \\
\varphi_{u \square v}(x \square y)=\tan ^{-1} \frac{\operatorname{Im}\left(G_{u \square v}(x \square y)\right)}{\operatorname{Re}\left(G_{u \square v}(x \square y)\right)}
\end{gathered}
$$

And

$$
\Delta \varphi_{u \square v}(x \square y)=\cos \left(\varphi_{u \square v}(x \square y)-\overline{\varphi_{v}}(x \square y)\right)-\left|\sin \left(\varphi_{u \square v}(x \square y)-\overline{\varphi_{v}}(x \square y)\right)\right|
$$

Where $\varphi_{u \square v}(x \square y)$ denotes the phase angle of the Gabor filter (with a center frequency $f_{u}$ and orientation $\left.\theta_{v}\right)$ at the spatial location $(x \square y)$, while $\overline{\varphi_{v}}(x \square y)$ represents the mean phase angle at the v-th orientation [39].

\section{B) Features based on intrinsic mode functions}

The aim of this step is to obtain features from intrinsic mode functions (IMFs). The empirical mode decomposition approach is a data dependent analysis which decomposes nonlinear and nonstationary signals to symmetrical components, which are known as intrinsic mode functions. (IMFs) [42]. It should be noted that, the IMFs obtained from the EMD method satisfy two following conditions:

1- Number of maxima and number of minima should have difference of at most one.

2- The mean value of two envelopes, one formed by connecting local maxima and another formed by connecting local minima of the signal should be zero.

IMFs can be derived from a signal $\mathrm{s}(\mathrm{t})$ using iterative process known as sifting process, explained by the following steps [41]:

1- Extraction of local maxima and local minima from signal $\mathrm{s}(\mathrm{t})$.

2- Obtaining the envelope $\varepsilon_{\max }(t)$ and $\varepsilon_{\min }(t)$ by connecting all the maxima and minima respectively.

3- Computing the average of $\varepsilon_{\max }(t)$ and $\varepsilon_{\min }(t)$ as:

$$
\alpha(t)=\frac{\varepsilon_{\max }(t)+\varepsilon_{\min }(t)}{2}
$$

4- Extraction of $\mathrm{d}(\mathrm{t})$ from $\mathrm{s}(\mathrm{t})$ as:

$$
d(t)=s(t)-\alpha(t)
$$

5- Whether the $\mathrm{d}(\mathrm{t})$ fulfils the above mentioned conditions for IMF or not.

6- Repeat steps $1-5$, for $d(t)$ until it satisfies the conditions for IMF.

Once the IMF is obtained, define $\operatorname{IMF}_{1}(t)=d(t)$. Now a residual signal $\mathrm{g}(\mathrm{t})$ can be obtained as $g(t)=s(t)-I M F_{1}(t)$, which serves as the new signal to extract the next IMF by applying sifting process. The sifting process is repeated until the residual obtained becomes monotonic function which means that no more IMF can be extracted. 

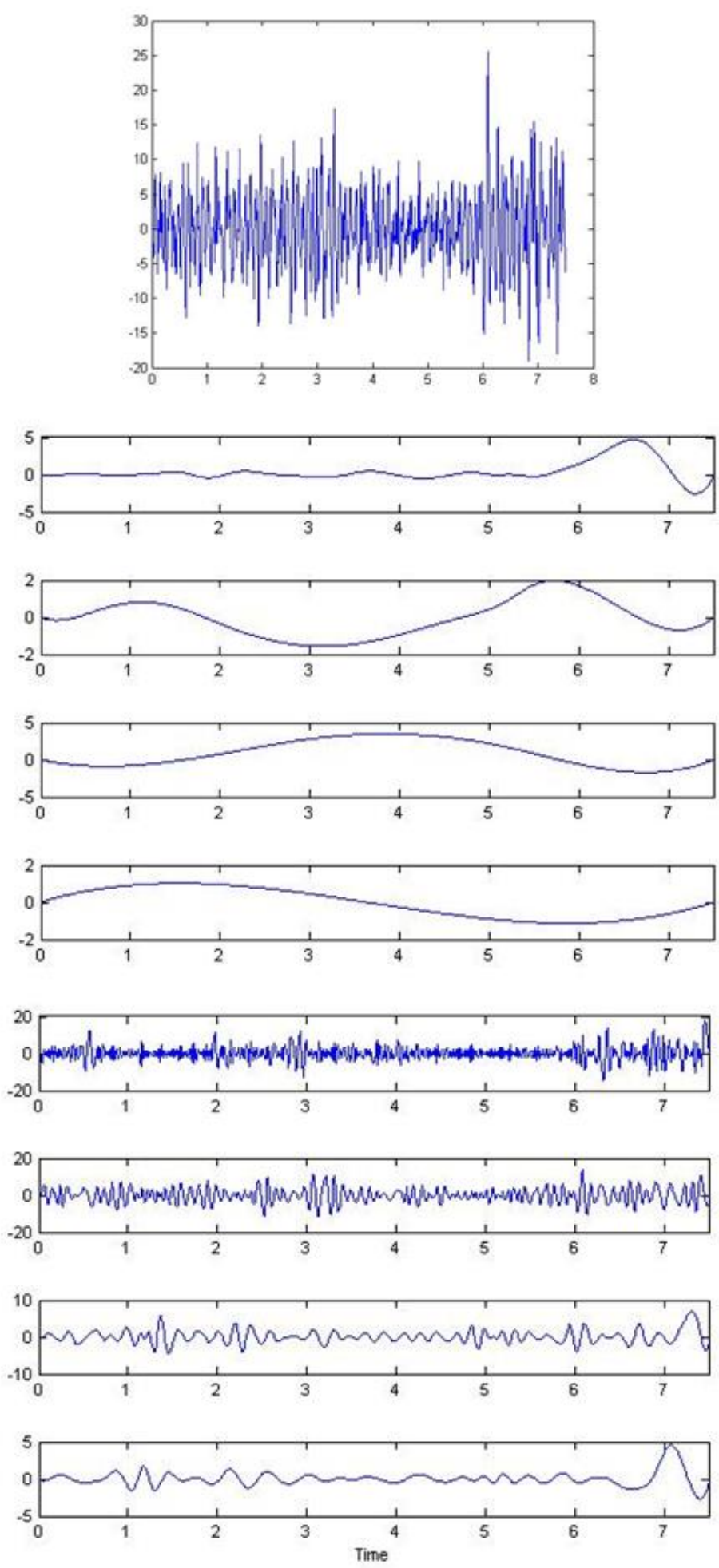

Figure 2. Sample signal of channel P7 and its IMFs 
Finally, the signal $\mathrm{s}(\mathrm{t})$ can be represented as the sum of IMFs and a residual [41]:

$$
s(t)=\sum_{i=1}^{M} I M F_{i}(t)+R(t)
$$

Where $M$ is number of extracted IMFs and $R(t)$ is residual. As an example Fig. 2 shows the IMFs of one channel data, where the horizontal and vertical axes represent time and magnitude of each signal, respectively.

In this study, for each signal just 3 primarily of the intrinsic mode functions are calculated and then the features are extracted from them. For each calculated IMFs, five characteristics including 1) maximum frequency b) center frequency c) Root Mean Square d) entropy and e) variance are computed.

\section{a) Maximum frequency}

IMF maximum frequency is considered as the frequency in which $95 \%$ of energy of the IMF signal is available before it. In this study, the first and second IMF maximum frequencies are calculated as features for each channel. For example, with the choice of 7 channels, 14 elements of the feature vector are related to the frequency of maximum frequency of IMFs.

\section{b) Central frequency}

The central frequency for the IMFs is defined as the frequency in which 50\% energy of IMF signals is available before it. In this study, only the central frequency of first IMF for each channel is intended as the feature.

\section{c) Entropy}

Entropy is a statistical model of the signal which can also provide physical information. Neurophysiological evidences show that it is possible to estimate the information from entropy as a measure of cortex performance. The change in entropy of EEG signals shows a real change in the function of the cerebral cortex. So, entropy, besides on a statistical measure of EEG pattern, in some cases, can reflect cortical information within the brain [10]. In this study, Shannon entropy is calculated to extract the IMFs features.

\section{d) Root Mean Square (RMS)}

Generally, the root mean square of the signal actually represents the average power signal. In this study, the root mean square of IMFs is used as extracted features. In this study, we used first three IMFs for root mean square.

\section{e) Variance}

In probability theory and statistics, variance is a measure of dispersion. In this study, the statistical data was used for extraction of features from the IMF. Again, like the entropy and root mean square, the 3 IMFs data are used to extract features from each channel which are 3 features belonging to variance for each channel.

\section{C) Production of feature vectors}

By this point, two sets of features have been extracted: the first set is related to applying Gabor wavelet transform on image. This set contains magnitude features such as mean and energy and also phase characteristics as OGPCP. The second set the features obtained by the intrinsic mode functions. The extracted features of this set are maximum and central frequency, entropy, root mean square and variance, as mentioned above. 


\subsection{Classification}

At this stage, the appropriate classification algorithm is applied to improve the accuracy of emotion recognition in accordance with the input feature vector compared to other existing methods. So, in this step, the goal is to find appropriate classifier for the generated feature vector.

SVM is a machine learning algorithm which is simple and has a high detection speed and accuracy. The idea of support vector machine is to find optimized hyper planes with maximum margin of separation.

In this research, the genetic optimization algorithm is used to find the optimized hyper planes. According to our knowledge on the emotions of EEG signals, optimized support vector machine has not been used. So, in addition to the innovations presented for feature extraction step, also, there is a new idea in the classification step, which is use of multi-class SVM optimized with genetic algorithm.

SVM has decisive parameters; some of them depend on the type of kernel function chosen for SVM algorithm. The kernel functions map the data, which is not separable linearly in its original space, to other space with more dimensions and separable linearly. There are some common kernel functions introduced, like Radial basis functions (RBF), sigmoid, linear, etc. In this study, the RBF kernel function is used. Choosing this function, two parameters should be determined which are called gamma and $\mathrm{C}$. These values have important influence on performance of the classifier. The effect of these two parameters on accuracy of classification is shown in table 3 .

Table3.Tthe effect of SVM parameters on classification accuracy

\begin{tabular}{|l|c|c|c|c|}
\hline Gamma $\left(\times 10^{-5}\right)$ & \multicolumn{2}{|c|}{4} & \multicolumn{2}{c|}{7} \\
\hline C & 9 & 10 & 9 & 10 \\
\hline Accuracy (\%) & 91.16 & 89.09 & 93.86 & 91.08 \\
\hline
\end{tabular}

\subsection{Cross Validation}

Based on a technique called cross-validation, the training data is divided into k subsets. $\mathrm{k}-1$ subsets are used for training and the unused one is used to evaluate the performance. This action is repeated $\mathrm{k}$ times and each time one new subset is considered for evaluation. In this process, all training data are once used for evaluation. We used 10-fold cross validation.

\section{Results}

In this experiment, an emotion representation model based on the valence-arousal model is adopted. Each dimension has ranging from 1 to 9. We segmented the Valence Arousal (VA) space to four quadrants according to the ratings. LALV, HALV, LAHV, and HAHV denote low arousal/low valence, high arousal/low valence, low arousal/high valence, and high arousal/high valence, respectively. The EEG data is then labeled according to participants' ratings of valence and arousal. The segmentation of the Valence Arousal (VA) space to four quadrants shows four general emotional states: happy, sad, exiting and hate.

The original EEG data of DEAP dataset is down sampled to $128 \mathrm{~Hz}$ and preprocessed with a band pass frequency filter $4.0-45.0 \mathrm{~Hz}$, also, the EOG artifacts are removed. So, we just extracted the mentioned Gabor and IMF features from the EEG data without any preprocessing. Then, the Multi Class SVM classifier with RBF kernel optimized by genetic algorithm is selected to classify the 
extracted features into four classes. To use the entire dataset for training and testing the classifier, a 10 -fold cross-validation scheme is adopted. All experiments are performed with 10-fold crossvalidation and the performance of the classification is evaluated through the classification accuracy rate.

Table 4 shows the effect of two different selected groups of EEG channel signals on emotion classification accuracy for 4 aforementioned classes. The experiments are achieved by two different Gabor filters, one is the filter with four scales and six orientations $(4 \times 6)$ and the other is the filter with five scales and eight orientations $(5 \times 8)$. The first group of channels contains P7 ‘P3 and $\mathrm{PZ}$ and the second group includes $\mathrm{P} 7$ ‘P3, PZ, PO3, C4, O1 and CP2.

Table4. Classification accuracy for two selected groups of channels and different Gabor filter sizes

\begin{tabular}{|c|c|c|}
\hline $\begin{array}{c}\text { Number of selected } \\
\text { channels }\end{array}$ & Gabor filter size & Classification accuracy (\%) \\
\hline \multirow{2}{*}{3} & $4 \times 6$ & 93.86 \\
\cline { 2 - 3 } & $5 \times 8$ & 90.28 \\
\hline \multirow{2}{*}{7} & $4 \times 6$ & 93.74 \\
\cline { 2 - 3 } & $5 \times 8$ & 90.41 \\
\hline
\end{tabular}

Choosing more channels and longer Gabor filters causes larger feature vector, but table 4, shows that the length of the feature vector is not directly proportional to the performance of the system. In this sense, sometimes with extending the feature vector length, the performance of detection accuracy is become even lower. So, we can conclude that the efficiency of the system performance is related to the effective extracted features, and not to the number of extracted features. On the other hand, the length of the feature vector is less, the greater the processing speed can result. This table shows that with selecting just 3 channels, named P7 ‘P3 and PZ and the Gabor filter with four scales and six orientations $(4 \times 6)$ provides effective feature vector that results better classification accuracy. In this case, the length of the feature vector was 264.

For fair comparison between different methods of EEG emotion recognition, some factors must be identical between them. Since different datasets sampled by different frequencies provide different information for the recognition system, it is needed the input signal should be identical. In addition, different emotion stimuli cause different level of reaction in different persons. The second factor is the number of classes which has to be the same.

Table 5, shows the comparison between the proposed method and several recent introduced methods. As shown, the proposed method is able to significantly improve the EEG emotion detection accuracy. In this table, only the reported method in [2] used the DEAP dataset as input, as same as the proposed method. It is obvious that the proposed algorithm enhances the accuracy of emotion detection compared to other algorithms. Table 6 , also, demonstrates the confusion matrix of the four aforementioned emotional states by the proposed method. 
Table5. Classification accuracy for two selected groups of channels and different Gabor filter sizes

\begin{tabular}{|c|c|c|c|}
\hline authors & Year published & $\begin{array}{c}\text { Classification } \\
\text { algorithm }\end{array}$ & $\begin{array}{c}\text { Classification } \\
\text { accuracy (\%) }\end{array}$ \\
\hline $\begin{array}{c}\text { Bajaj and Pachori } \\
\text { [27] }\end{array}$ & 2015 & SVM & 84.79 \\
\hline Zheng et al. [2] & 2016 & SVM & 69.67 \\
\hline Li and Lu [43] & 2009 & LDA, KNN & 83.04 \\
\hline Lin et al. [44] & 2010 & SVM & 66.29 \\
\hline Wang et al.[7] & 2011 & SVM & 93.86 \\
\hline Proposed method & 2016 & Evolutionary SVM & \multicolumn{2}{|c|}{} \\
\hline
\end{tabular}

Table6. Confusion matrix for Classification of four emotional states by the proposed method (\%)

\begin{tabular}{|c|c|c|c|c|}
\hline Emotions & Happy & Sad & Exiting & Hate \\
\hline Happy & $\mathbf{9 5 . 3}$ & 0.3 & 2.8 & 1.6 \\
\hline Sad & 0.4 & $\mathbf{9 4 . 8}$ & 1.2 & 3.6 \\
\hline Exiting & 4.35 & 2.2 & $\mathbf{9 1 . 3 5}$ & 2.1 \\
\hline Hate & 1.1 & 2.2 & 2.7 & $\mathbf{9 4 . 0}$ \\
\hline Accuracy(\%) & 95.3 & 94.8 & 91.35 & 94.0 \\
\hline
\end{tabular}

\section{Conclusion}

This study was conducted to improve the accuracy of detecting the emotions based on the EEG signals. In general, studies with this subject have several basic steps. The first step is the preparation of appropriate data. In this step, the DEAP database was used to validate the proposed algorithm. The second step is extracting the features which were proposed by innovative features extracted based on filtering the data with Gabor filters and also, applying the intrinsic mode functions. The experimental results show that selection of 7.5 seconds in length for input data and 3 channels provide acceptable results. The last step is choosing suitable classification algorithm. We found that the multiclass SVM algorithm enhanced by the Genetic evolutionary algorithm provides better performance.

At the end, it was shown a dramatic improvement in accuracy of diagnostic emotions. $93.86 \%$ is detection accuracy of the proposed algorithm which is able to detect 4 different emotional states based on the EEG signals. 


\section{References}

[1] Muhl C, Allison B, Nijholt A, \& Chanel G. "A survey of affective brain computer interfaces: principles, state-of-the-art, and challenges," Brain-Computer Interfaces, vol. 1, no. 2, pp.66-84, 2014, doi: 10.1080/2326263X.2014.912881.

[2] Zheng W.L, Zhu J.Y and Lu B.L,'Identifying Stable Patterns over Time for Emotion Recognition from EEG," Human-Computer Interaction (cs.HC). arXiv preprint arXiv:1601.02197, 2016.

[3] Chanel G, Ciftci K, Monta J.C, Savran A, Viet L.H, Akarun L, Caplier A, Rombaut M and Sankur B," Emotion detection in the loop from brain signals and facial images", in Proceedings of the eNTERFACE, 2006 workshop, doi:10.1109/CIBEC.2008.4786037.

[4] Chen L, Mao X, Xue Y and Cheng LL, "Speech emotion recognition: Features and classification models" Digital Signal Processing, vol. 22, no.6, pp. 1154-1160, 2012, doi: 10.1016/j.dsp.2012.05.007.

[5] Jenke R, Peer A and Buss M," Feature Extraction and Selection for Emotion Recognition from EEG” IEEE Trans. Effective Computing, vol. 5, no.3, pp.327-339, 2014, doi: Jenke R, Peer A and Buss M," Feature Extraction and Selection for Emotion Recognition from EEG" IEEE Trans. Effective Computing, vol. 5, no.3, pp. 327-339, 2014, doi: 10.1109/TAFFC.2014.2339834.

[6] Liu Y and Sourina O. "Real-time fractal-based valence level recognition from EEG" Trans. Computational Science XVIII. 7848, pp.101-120, 2013, doi: 10.1007/978-3-642-38803-36.

[7] Wang X, Nie D and Lu B. "EEG-based emotion recognition using frequency domain features and support vector machines", International Conf. on Neural Information Processing (ICONIP), pp.734-743, 2011, doi: 10.1007/978-3-642-24955-6_87.

[8] Murugappan M, Rizon M, Nagarajan R and Yaacob S. “ Inferring of human emotional states using multichannel EEG” European journal of Scientific Research, vol. 48, no.2, pp.281-299, 2010.

[9] Takahashi K. "Remarks on emotion recognition from multi-modal bio-potential signals" International Conf. on Industrial Technology (ICIT), pp.1138-1143, 2004, doi: 10.1007/978-3642-29752-6_19.

[10] Sleigh JW, Olofsen E, Dahan A, Goede J and Steyn-Ross A. "Entropies of the EEG: the effects of general anesthesia" 5th International Conf. on Memory, Awareness and Consciousness, pp.1-3, 2001.

[11] Hjorth B. "EEG analysis based on time domain properties. Electroencephalography and Clinical" Neurophysiology, vol. 29, no. 3, pp. 306-310, 1970, doi: 10.1016/00134694(70)90143-4.

[12] Ansari-asl K, Chanel G and Pun T. " A channel selection method for EEG classification in emotion assessment based on synchronization likelihood," 15th European Signal Processing Conf. (EUSIPCO), pp. 1241-1245, 2007.

[13] Horlings R, Datcu D and Rothkrantz L." Emotion recognition using brain activity" International Conf. on Computer Systems and Technologies (Comp Sys Tech), pp.1-6, 2008, doi: 10.1145/1500879.1500888.

[14] Liu Y and Sourina O. "EEG-based subject-dependent emotion recognition algorithm using fractal dimension" 2014 IEEE International Conf. on Systems, Man and Cybernetics (SMC), pp. 3166 - 3171, 2014, doi: 10.1109/SMC.2014.6974415.

[15] Kroupi E, Yazdani A and Ebrahimi T. "EEG correlates of different emotional states elicited during watching music videos" International Conf. on Affective Computing and Intelligent Interaction (ACII), Springer. 6975, pp. 457-466, 2011, doi: 10.1007/978-3-642-24571-8_58. 
[16] Petrantonakis PC and Hadjileontiadis LJ. "Emotion recognition from EEG using higher order crossings" IEEE Trans. on Information Technology in Biomedicine, vol. 14, no.2, pp.186197, 2010, doi: 10.1109/TITB.2009.2034649.

[17] Petrantonakis PC and Hadjileontiadis LJ. "A novel emotion elicitation index using frontal brain asymmetry for enhanced EEG based emotion recognition" IEEE Trans. on Information Technology of Biomedical, vol. 15, no.5, pp. 737-46, 2011, doi: 10.1109/TITB.2011.2157933.

[18] Hosseini S, Khalilzadeh M, Naghibi-Sistani M and Niazmand V. " Higher Order Spectra Analysis of EEG Signals in Emotional Stress States" IEEE International Conf. on Information Technology and Computer Science, pp. 60-63, 2010, doi: 10.1109/ITCS.2010.21.

[19] Nie D, Wang XW, Shi LC and Lu BL. "EEG-based Emotion Recognition during Watching Movies" IEEE International Conf. on Neural Engineering, pp.667-670, 2011.

[20] Liu Y and Sourina O. "EEG-based dominance level recognition for emotion-enabled interaction" IEEE International Conf. on Multimedia and Expo., pp.1039-1044, 2012, doi: 10.1109/ICME.2012.20

[21] Reuderink B, Muh C and Poel M. "Valence, arousal and dominance in the EEG during game play", International Journal of Autonomous and Adaptive Communications Systems, vol. 6, no. 1, pp. 45-62, 2013, doi: 10.1504/IJAACS.2013.050691.

[22] Rozgi'c V, Vitaladevuni S and Prasad R. "Robust EEG emotion classification using segment level decision fusion" International Conf. on Acoustics, Sound and Signal Processing (ICASSP), pp.1286-1290, 2013.

[23] Soleymani M, Kolestra S, Patras I and Pun T. "Continuous emotion detection in response to music videos" IEEE International Conf. on Automatic Face \& Gesture Recognition, pp.803808, 2011.

[24] Stelios K and Hadjidimitriou LJ. "Toward an EEG-based recognition of music liking using time-frequency analysis" IEEE Trans. on Biomedical Engineering, vol. 59, no. 12, pp.34983510, 2012, doi: 10.1109/TBME.2012.2217495.

[25] Murugappan M, Nagarajan R and Yaacob S. "Classification of human emotion from EEG using discrete wavelet transform" Journal of Biomedical Science and Engineering, vol.3, no.4, pp. 390-396, 2010, doi: 10.4236/jbise.2010.34054.

[26] Hadjidimitriou SK and Hadjileontiadis LJ. "Toward an EEG-based recognition of music liking using time-frequency analysis" IEEE Trans. on Biomedical Engineering, vol. 59, no.12, pp.3498-510, 2012, doi: 0.1109/TBME.2012.2217495.

[27] Bajaj V and Pachori RB. "Detection of Human Emotions Using Features Based on the Multiwavelet Transform of EEG Signals", In: Hassanien (editor): Brain-Computer Interfaces. Springer International Publishing. Switzerland. pp.215-240, 2015, doi: 10.1007/978-3-31910978-7_8.

[28] Liu Y and Sourina O. "Real Time subject dependent EEG based Emotion Recognition Algorithm” Trans. on Computer Science XXIII, Springer- Verlag Berlin Heidelberg, vol. 32, no.3, pp. 199-223, 2014.

[29] Park MS, Oh HS and Jeong H. "EEG based emotion recognition during emotionally evocative films" International Winter Workshop on Brain Computer Interface (BCI), pp.18-20, 2013, doi: 10.1109/IWW-BCI.2013.6506629.

[30] Kwon M, Kang JS and Lee M. "Emotion classification in movie clips based on 3D fuzzy gist and EEG signal analysis" International Winter Workshop on Brain Computer Interface (BCI), pp. 67-68, 2013 .

[31] Chung SY and Yoon HJ. "Affective classification using Bayesian classifier and supervised learning" 12th International Conf. on Control, Automation and System (ICCAS), pp.17681771, 2012.

[32] Hosseini SA and Khalilzadeh MA. "Emotional Stress Recognition System using EEG and Psychophysiological Signals: Using New Labeling Process of EEG Signals in Emotional 
Stress State" Proceedings of the IEEE, The International Conf. on Biomedical Engineering and Computer Science (ICBECS), pp.90-95, 2010.

[33] Khosrowabadi R and Rahman AW. "Classification of EEG correlates on emotion using features from Gaussian Mixtures of EEG Spectrogram" International Conf. on Information and Communication Technology for the Muslim World (ICT4M), pp.13-14, 2010.

[34] Petrantonakis PC and Hadjileontiadis, LJ. "Emotion recognition from EEG using higher order crossings" IEEE Trans. on Information Technology in Biomedicine, vol.14, no.2, pp.186-197, 2014, doi: 10.1109/TITB.2009.2034649.

[35] Koelstra S, Muhl C, Soleymani M, Lee JS, Yazdani A, Ebrahimi T, Pun T, Nijholt A and Patras I. "Deap: A database for emotion analysis; using physiological signals" IEEE Trans. on Affective Computing. Vol. 3, no.1, pp.42-55, 2012, doi: 10.1109/T-AFFC.2011.15.

[36] Vijayan AE, Sen D and Sudheer AP. "EEG-based Emotion Recognition using Statistical measures and Auto-regressive modeling" IEEE International Conf. on Computational Intelligence \& Communication Technology, pp.587 - 591, 2015.

[37] Ansari- asl K, Chanel G and Pun T. "A channel selection method for EEG classification in emotion assessment based on synchronization likelihood" 15th European Signal Processing Conf. (EUSIPCO), pp.1241-1245, 2007.

[38] Struc V and Pavesic N. "Gabor-based Kernel-partial-least-squares Discrimination Features for Face Recognition” Informatica (Vilnius), vol. 20, no. 1, pp.115-138, 2009.

[39] Struc V and Pavesic N. "From Gabor Magnitude to Gabor Phase Features: Tackling the Problem of Face Recognition under Severe Illumination Changes" In: M. Oravec (editor): Face Recognition, In-Tech, Vienna. pp. 215-238, 2010, doi: 10.5772/8938.

[40] Zhang B, Shan S, Chen X. and Gao W. "Histogram of gabor phase patterns (hgpp): A novel object representation approach for face recognition" IEEE Trans. on Image Processing, vol.16, no.1, pp.57-68, 2007, doi: 10.1109/TIP.2006.884956.

[41] Huang NE, Shen Z, Long SR, Wu MC, Shih HH, Zheng Q, Yen NC, Tung CC and Liu HH. "The empirical mode decomposition and the Hilbert spectrum for nonlinear and nonstationary time series analysis" Proceedings of the Royal Society of London. Series A: Mathematical, Physical and Engineering Sciences, pp. 903-995, 1998, doi: 10.1098/rspa.1998.0193.

[42] Sharma R and Pachori RB. "Classification of epileptic seizures in EEG signals based on phase space representation of intrinsic mode functions" Expert Systems with Applications, vol. 42, no.3, pp.1106-1117, 2015, doi: 10.1016/j.eswa.2014.08.030.

[43] Li M and Lu BL. " Emotion classification based on gamma band EEG” Conf. Proceedings: IEEE Engineering in Medicine and Biology Society, pp.1323-1326, 2009, doi: 10.1109/IEMBS.2009.5334139.

[44] Lin YP, Wang CH, Wu TL, Jeng SK and Chen JH. "EEG-based emotion recognition in music listening" IEEE Trans. on Biomedical Engineering, vol.57, no.7, pp.1798-806, 2010, doi: 10.1109/TBME.2010.2048568. 